\title{
Growth and Yield Response of Mungbean Plants to Colonization of Arbuscular Mycorrhizal Fungi (AMF) (Glomus macrocarpon) and Phosphorus Amendments
}

\author{
Amandeep Singh*, Daljeet Singh and Narinder Singh
}

Senior Plant Pathologist, College of Agriculture, Punjab Agricultural University,
Ludhiana-141004, India

*Corresponding author

\section{A B S T R A C T}

\begin{tabular}{l} 
Ke y w o r d s \\
AM Fungi, \\
colonization, \\
$\begin{array}{l}\text { Glomus } \\
\text { macrocarpon, } \\
\text { Mungbean, Spore } \\
\text { population }\end{array}$ \\
\hline Article Info \\
$\begin{array}{l}\text { Accepted: } \\
\text { 04 November } 2019 \\
\text { Available Online: } \\
\text { 10 December } 2019\end{array}$ \\
\hline
\end{tabular}

Arbuscular mycorrhizal fungi (AM fungi) have been found associated with a wide variety of agricultural and horticultural crops, but in legumes it occurs widely. The experiments were conducted during spring season 2017 under pot house conditions. The effect of inoculation of AM Fungi colonization and $\mathrm{P}$ amendments on growth and yield of Mungbean plants was investigated. The results revealed the overall increase in plant growth parameters and yield of Mungbean plants. In AM Fungi inoculated pots, the root as well as leaf surface area were also enhanced significantly due to the colonization of Glomus macrocarpon (AM Fungi) as compared to uninoculated plots. Among three levels of Phosphorus; 0, 40 and $80 \mathrm{mg} / \mathrm{kg}$ soil, the maximum increase in root and shoot length, their fresh and dry weight was recorded at $40 \mathrm{mg} \mathrm{P} / \mathrm{kg}$ soil in 15, 30, 45 and 60 days old Mungbean plants as compared to 80 and $0 \mathrm{mg} \mathrm{P} / \mathrm{kg}$ soil levels. The number of leaves and pods per plant, their fresh and dry weight was also found maximum at $40 \mathrm{mg} \mathrm{P} / \mathrm{kg}$ soil level. Maximum increase in plant growth and yield of Mungbean plants were recorded in the pots combined inoculated with Glomus macrocarpon and phosphorus. It is suggested that Arbuscular Mycorrhizal Fungi have the potential to act as bio-fertilizer.

\section{Introduction}

Mycorrhihza refers to the mutualistic, symbiosis between fungi and roots of higher plants. The vesicular Arbuscular mycorrhizal fungi is very common and old among all mycorrhizal types (Mosse et al., 1981). The VAM fungus occur widely in legumes (Bargali, 2011) and number of forage crops under different ecosystems (Souchie et al., 2006). Mungbean is an important pulse crop as it supplies 25 per cent protein. So the interaction of mycorrhizal fungi with roots of Mungbean plants is very important. The mycorrrhizal colonization is facilitating the efficient use of nutrients and water up take by plants especially in infective soil (Harley and Smith, 1983). Symbiotic association between VAM and roots of plants enhanced the nutrient absorbing area of roots along with improvement in efficiency of nutrient absorption (Kadian et al., 2014). In addition, 
Arbuscular mycorrhizal fungus is known to protect the plants against several soil borne diseases (Davis and Menge, 1980; Singh et al., 2017). VAM fungus improves the soil structure through the capacity to bind soil particles into crumbs, which is required soil structure for growth of plants (Manke et al., 2008). Researchers in the last few decades have established that VAM improve the plant growth and have potential to act as plant biofertilizer.

\section{Materials and Methods}

\section{Treatments}

Different experiments were conducted to observe the impact of Arbuscular Mycorrhizal fungus on growth and yield parameters of mungbean plants in pots using sterilized soil. The experiments comprised of five treatments namely, 1. Arbuscular Mycorrhizal fungus (AMF) alone 2. Pathogen (Macrocphomina phaseolina (Tassi) Goid) alone 3. AMF/Pathogen (AMF was applied at the time of sowing and pathogen was applied after one week of sowing) 4. Pathogen/AMF (Pathogen was applied at time of sowing and AM F was applied after 5 days of sowing) 5 . Uninoculated Control (without any treatment) and designed at 3 levels of phosphorus in soil i. e. (1) Control $\left(\mathrm{P}_{0}\right)(2)\left(\mathrm{P}_{40}\right)(3) 80 \mathrm{mg}$ 'P'/ kg soil $\left(\mathrm{P}_{80}\right)$ Total seven plants maintained per pot and treatments were replicated thrice.

Cleaning and staining of roots were done by using method of Philips and Haymann (1970). The mycorrhizal colonization was measured by using the method of Biermann and Linderman (1981). Extraction of spores of AM fungi was done by using wet sieving and decanting technique (Gredmann and Nicolson 1963). Observations regarding the percentage colonization, spore population and plant growth parameters were taken after 15, 30, 45 and 60 days of sowing. After taking fresh weight, the plant parts were kept separately in the oven at $80^{\circ} \mathrm{C}$ for 48 hours for complete drying of samples in order to record their dry weight.

\section{Results and Discussion}

\section{Mycorrhizal colonization and spore population}

The inoculation of Glomus macrocarpon showed the maximum colonization at $40 \mathrm{mg}$ ' $\mathrm{P}$ '/ $\mathrm{kg}$ soil $(36.60,52.50,69.10$ and 78.00 per cent after 15, 30, 45 and 60 days of sowing) as compared to $31.25,44.60,62.00$ and 72.20 per cent colonization at $80 \mathrm{mg}$ ' $\mathrm{P}$ '/ $\mathrm{kg}$ soil level. There is direct relationship between spore population and mycorrhizal colonization. The spore population was $400,593,785$ and 950 per $100 \mathrm{~g}$ soil at $40 \mathrm{mg}$ 'P'/ $\mathrm{kg}$ soil as compared to $350,480,710$ and 825 spores per $100 \mathrm{~g}$ soil at $80 \mathrm{mg}$ ' $\mathrm{P}$ '/ $\mathrm{kg}$ soil level. It was found that with increase in mycorrhizal colonization is directly proportional to the age of the plant. At initial stages the colonization is very less, but with age of the plants the colonization increased. There is no colonization observed in root samples collected from the uninoculated plants.

\section{Effect on plant growth parameters}

The plant growth responses with respect to root, shoot length, fresh and dry weight along with number of leaves their fresh and dry weight were significantly higher in Glomus macrocarpon inoculated plants at all level of ' $\mathrm{P}$ ' in soil as compared to uninoculated plants (Table 3 to 6). It was found that the application of phosphorus at different levels significantly affect the colonization as well as growth of plants. Among the three levels of $\mathrm{P}$, the plants were more responsive to $40 \mathrm{mg}$ ' $\mathrm{P}$ '/ $\mathrm{kg}$ soil level as compared to the $80 \mathrm{mg} \mathrm{P} / \mathrm{kg}$ soil and control plants (Fig. 1). Rabie (2005) studied that the dry weight as well as plant 
height of mungbean plants colonized by $G$. plants. Similar results were found by Sohrabi clarum was significantly higher than untreated et al., (2015).

Fig. 1 Comparison of Plant growth of AM inoculated and un-inoculated plants at different levels of $\mathrm{P}$

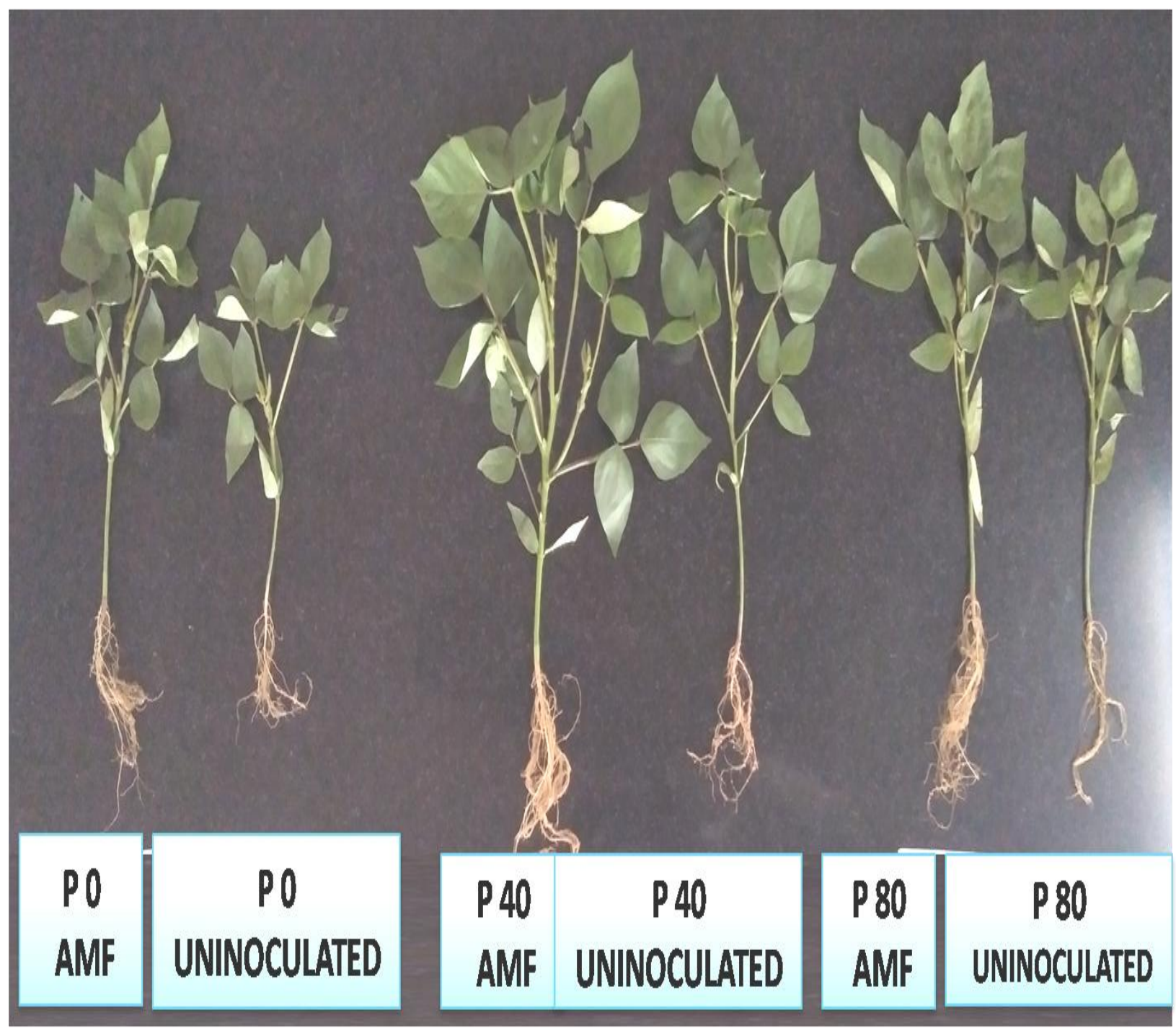




\section{Int.J.Curr.Microbiol.App.Sci (2019) 8(12): 3039-3048}

Table.1 Effect of Glomus macrocarpon inoculation and 'P' amendment on Arbuscular mycorrhizal colonization in roots of Mungbean

\begin{tabular}{|c|c|c|c|c|c|c|c|c|c|c|c|c|c|c|c|c|}
\hline \multirow{4}{*}{ Treatment } & \multicolumn{16}{|c|}{ Observations after Days } \\
\hline & \multicolumn{4}{|c|}{15} & \multicolumn{4}{|c|}{30} & \multicolumn{4}{|c|}{60} & \multicolumn{4}{|c|}{90} \\
\hline & \multirow{2}{*}{$\begin{array}{l}\text { Coloni- } \\
\text { zation } \\
(\%)\end{array}$} & \multicolumn{3}{|c|}{ Mycorrhizal Structures } & \multirow{2}{*}{$\begin{array}{l}\text { Coloni- } \\
\text { zation } \\
(\%)\end{array}$} & \multicolumn{3}{|c|}{ Mycorrhizal Structures } & \multirow{2}{*}{$\begin{array}{c}\text { Coloni } \\
\text { zation } \\
(\%)\end{array}$} & \multicolumn{3}{|c|}{ Mycorrhizal Structures } & \multirow{2}{*}{$\begin{array}{c}\text { Coloni- } \\
\text { Zation } \\
(\%)\end{array}$} & \multicolumn{3}{|c|}{ Mycorrhizal Structures } \\
\hline & & $\begin{array}{l}\text { Myce- } \\
\text { lium }\end{array}$ & $\begin{array}{l}\text { Arbus- } \\
\text { cules }\end{array}$ & $\begin{array}{l}\text { Vesi- } \\
\text { cles }\end{array}$ & & $\begin{array}{l}\text { Myce- } \\
\text { lium }\end{array}$ & $\begin{array}{l}\text { Arbus- } \\
\text { cules }\end{array}$ & $\begin{array}{l}\text { Vesic- } \\
\text { les }\end{array}$ & & $\begin{array}{c}\text { Myce- } \\
\text { lium }\end{array}$ & $\begin{array}{l}\text { Arbu- } \\
\text { scules }\end{array}$ & $\begin{array}{l}\text { Vesic- } \\
\text { les }\end{array}$ & & $\begin{array}{c}\text { Myce- } \\
\text { lium }\end{array}$ & $\begin{array}{l}\text { Arbus- } \\
\text { cules }\end{array}$ & $\begin{array}{l}\text { Vesic- } \\
\text { les }\end{array}$ \\
\hline \multicolumn{17}{|l|}{ Main effects } \\
\hline $\mathbf{U}$ & $\begin{array}{c}00.00 \\
(00.00)\end{array}$ & - & - & - & $\begin{array}{c}00.00 \\
(00.00)\end{array}$ & - & - & - & $\begin{array}{c}00.00 \\
(00.00)\end{array}$ & - & - & - & $\begin{array}{c}00.00 \\
(00.00)\end{array}$ & - & - & - \\
\hline $\mathbf{I}$ & $\begin{array}{c}31.72 \\
(34.22)\end{array}$ & +++ & ++ & - & $\begin{array}{c}44.90 \\
(42.00)\end{array}$ & +++ & +++ & + & $\begin{array}{c}62.60 \\
(52.30)\end{array}$ & ++ & ++ & ++ & $\begin{array}{c}72.20 \\
(58.20)\end{array}$ & ++ & + & +++ \\
\hline C D at 5\% & 0.29 & & & & 0.26 & & & & 0.31 & & & & 0.22 & & & \\
\hline $\mathbf{P}_{0}$ & $\begin{array}{c}13.60 \\
(15.70)\end{array}$ & ++ & + & - & $\begin{array}{c}18.70 \\
(18.80)\end{array}$ & ++ & ++ & + & $\begin{array}{c}28.30 \\
(24.40)\end{array}$ & + & ++ & ++ & $\begin{array}{c}33.20 \\
(27.20)\end{array}$ & + & - & ++ \\
\hline $\mathbf{P}_{40}$ & $\begin{array}{c}18.30 \\
(18.60)\end{array}$ & ++ & + & - & $\begin{array}{c}26.20 \\
(23.20)\end{array}$ & ++ & ++ & + & $\begin{array}{c}34.50 \\
(28.12)\end{array}$ & + & ++ & ++ & $\begin{array}{c}39.00 \\
(31.00)\end{array}$ & + & - & +++ \\
\hline $\mathbf{P}_{80}$ & $\begin{array}{c}15.60 \\
(16.90)\end{array}$ & ++ & + & - & $\begin{array}{c}22.30 \\
(20.90)\end{array}$ & ++ & ++ & + & $\begin{array}{c}31.00 \\
(25.90)\end{array}$ & + & ++ & ++ & $\begin{array}{c}36.10 \\
(29.00)\end{array}$ & + & - & ++ \\
\hline C D at $5 \%$ & 0.36 & & & & 0.32 & & & & 0.38 & & & & 0.27 & & & \\
\hline \multicolumn{17}{|c|}{ Interaction effects } \\
\hline $\mathbf{P}_{0} \mathrm{U}$ & $\begin{array}{c}00.00 \\
(00.00)\end{array}$ & - & - & - & $\begin{array}{c}00.00 \\
(00.00)\end{array}$ & - & - & - & $\begin{array}{c}00.00 \\
(00.00)\end{array}$ & - & - & - & $\begin{array}{c}00.00 \\
(00.00)\end{array}$ & - & - & - \\
\hline $\mathbf{P}_{40} \mathbf{U}$ & $\begin{array}{c}00.00 \\
(00.00)\end{array}$ & - & - & - & $\begin{array}{c}00.00 \\
(00.00)\end{array}$ & - & - & - & $\begin{array}{c}00.00 \\
(00.00)\end{array}$ & - & - & - & $\begin{array}{c}00.00 \\
(00.00)\end{array}$ & - & - & - \\
\hline $\mathbf{P}_{80} \mathbf{U}$ & $\begin{array}{c}00.00 \\
(00.00)\end{array}$ & - & - & - & $\begin{array}{c}00.00 \\
(00.00)\end{array}$ & - & - & - & $\begin{array}{c}00.00 \\
(00.00)\end{array}$ & - & - & - & $\begin{array}{c}00.00 \\
(00.00)\end{array}$ & - & - & - \\
\hline $\mathbf{P}_{0} \mathbf{I}$ & $\begin{array}{c}27.20 \\
(31.40)\end{array}$ & ++ & ++ & - & $\begin{array}{c}37.50 \\
(37.73)\end{array}$ & +++ & +++ & + & $\begin{array}{c}56.70 \\
(48.80)\end{array}$ & ++ & ++ & +++ & $\begin{array}{c}66.40 \\
(54.50)\end{array}$ & ++ & + & +++ \\
\hline $\mathbf{P}_{40} \mathbf{I}$ & $\begin{array}{c}36.60 \\
(37.20)\end{array}$ & ++ & ++ & - & $\begin{array}{c}52.50 \\
(46.45)\end{array}$ & +++ & +++ & ++ & $\begin{array}{c}69.10 \\
(56.20)\end{array}$ & ++ & ++ & +++ & $\begin{array}{c}78.00 \\
(62.00)\end{array}$ & ++ & + & +++ \\
\hline $\mathbf{P}_{80} \mathbf{I}$ & $\begin{array}{c}31.25 \\
(33.90)\end{array}$ & ++ & ++ & - & $\begin{array}{c}44.60 \\
(41.80)\end{array}$ & +++ & +++ & ++ & $\begin{array}{c}62.00 \\
(51.90)\end{array}$ & ++ & ++ & +++ & $\begin{array}{c}72.20 \\
(58.10)\end{array}$ & ++ & + & +++ \\
\hline C D at $5 \%$ & 0.51 & & & & 0.46 & & & & 0.54 & & & & 0.38 & & & \\
\hline
\end{tabular}

$\mathrm{P}_{0}=$ No phosphorous application; $\mathrm{P}_{40}=40 \mathrm{mg}$ ` $\mathrm{P} / \mathrm{kg}$ soil; $\mathrm{P}_{80}=80 \mathrm{mg}$ ` $\mathrm{P} / \mathrm{kg}$ soil; U =Uninoculated; I = Inoculated; Figures in parentheses are arc sin transformed value of respective data; $+=$ Scanty $++=$ Moderate; $+++=$ Abundant 
Table.2 Effect of Glomus macrocarpon and P amendments on plant growth parameters of Mungbean after 15 days of sowing

\begin{tabular}{|c|c|c|c|c|c|c|c|c|c|c|c|}
\hline \multicolumn{12}{|c|}{15 DAYS } \\
\hline \multirow[b]{2}{*}{ Treatment } & \multicolumn{3}{|c|}{ Root } & \multicolumn{3}{|c|}{ Shoot } & \multicolumn{3}{|c|}{ Leaves } & \multirow{2}{*}{$\begin{array}{c}\text { Branches } \\
\text { Number }\end{array}$} & \multirow{2}{*}{$\begin{array}{l}\text { Nodu- } \\
\text { lation }\end{array}$} \\
\hline & $\begin{array}{c}\text { Length } \\
(\mathrm{cm})\end{array}$ & $\begin{array}{c}\text { Fresh } \\
\text { weight } \\
\text { (g) }\end{array}$ & $\begin{array}{c}\text { Dry } \\
\text { weight } \\
\text { (g) }\end{array}$ & $\begin{array}{c}\text { Length } \\
\text { (cm) }\end{array}$ & $\begin{array}{c}\text { Fresh } \\
\text { weight } \\
\text { (g) }\end{array}$ & $\begin{array}{c}\text { Dry } \\
\text { weight } \\
\text { (g) }\end{array}$ & Number & $\begin{array}{c}\text { Fresh } \\
\text { weight } \\
\text { (g) }\end{array}$ & $\begin{array}{c}\text { Dry } \\
\text { weight } \\
\text { (g) }\end{array}$ & & \\
\hline \multicolumn{12}{|l|}{ Main effects } \\
\hline $\mathbf{U}$ & 8.72 & 0.96 & 0.54 & 8.53 & 2.00 & 1.06 & 5.13 & 1.64 & 0.79 & 1.27 & - \\
\hline C D at $5 \%$ & 0.56 & 0.06 & 0.07 & 0.23 & 0.04 & 0.09 & 0.36 & 0.09 & 0.04 & 0.29 & \\
\hline $\mathbf{P}_{0}$ & 10.30 & 1.22 & 0.60 & 10.60 & 2.75 & 1.45 & 5.80 & 1.95 & 0.96 & 1.50 & + \\
\hline $\mathbf{P}_{40}$ & 14.20 & 2.24 & 1.26 & 14.60 & 5.35 & 2.70 & 7.60 & 4.00 & 2.03 & 2.25 & ++ \\
\hline $\mathbf{P}_{80}$ & 11.88 & 1.77 & 0.90 & 12.10 & 4.35 & 2.15 & 6.70 & 2.60 & 1.35 & 1.58 & + \\
\hline \multicolumn{12}{|c|}{ Interaction effects } \\
\hline $\mathbf{P}_{40} \mathbf{U}$ & 10.20 & 1.30 & 0.82 & 10.20 & 2.70 & 1.40 & 5.60 & 2.18 & 1.16 & 1.50 & - \\
\hline $\mathbf{P}_{\mathbf{8 0}} \mathbf{U}$ & 8.76 & 0.93 & 0.50 & 8.20 & 2.00 & 1.00 & 5.40 & 1.60 & 0.80 & 1.33 & - \\
\hline $\mathbf{P}_{0} \mathbf{I}$ & 13.40 & 1.80 & 0.90 & 14.00 & 4.20 & 2.10 & 7.20 & 2.76 & 1.50 & 2.00 & + \\
\hline $\mathbf{P}_{40} \mathbf{I}$ & 18.20 & 3.18 & 1.70 & 19.00 & 8.00 & 4.00 & 9.60 & 5.82 & 2.90 & 3.00 & ++ \\
\hline $\mathbf{P}_{80} \mathbf{I}$ & 15.00 & 2.60 & 1.30 & 16.00 & 6.70 & 3.30 & 8.00 & 3.60 & 1.90 & 1.83 & ++ \\
\hline C D at $5 \%$ & 0.98 & 0.11 & 0.12 & 0.41 & 0.07 & 0.15 & 0.63 & 0.16 & 0.07 & 0.36 & \\
\hline
\end{tabular}

$\mathrm{P}_{0}=$ No phosphorous application.; $\mathrm{P}_{40}=40 \mathrm{mg} ` \mathrm{P}$ ` $\mathrm{kg}$ soil; $\mathrm{P}_{80}=80 \mathrm{mg} ` \mathrm{P} ` \mathrm{~kg}$ soil; $\mathrm{U}=$ Uninoculated; $\mathrm{I}=$ Inoculated; + = Scanty; + + = Moderate; + + + = Abundant 
Table.3 Effect of Glomus macrocapon inoculation and P amendments on plant growth parameters of Mungbean 30 days after sowing

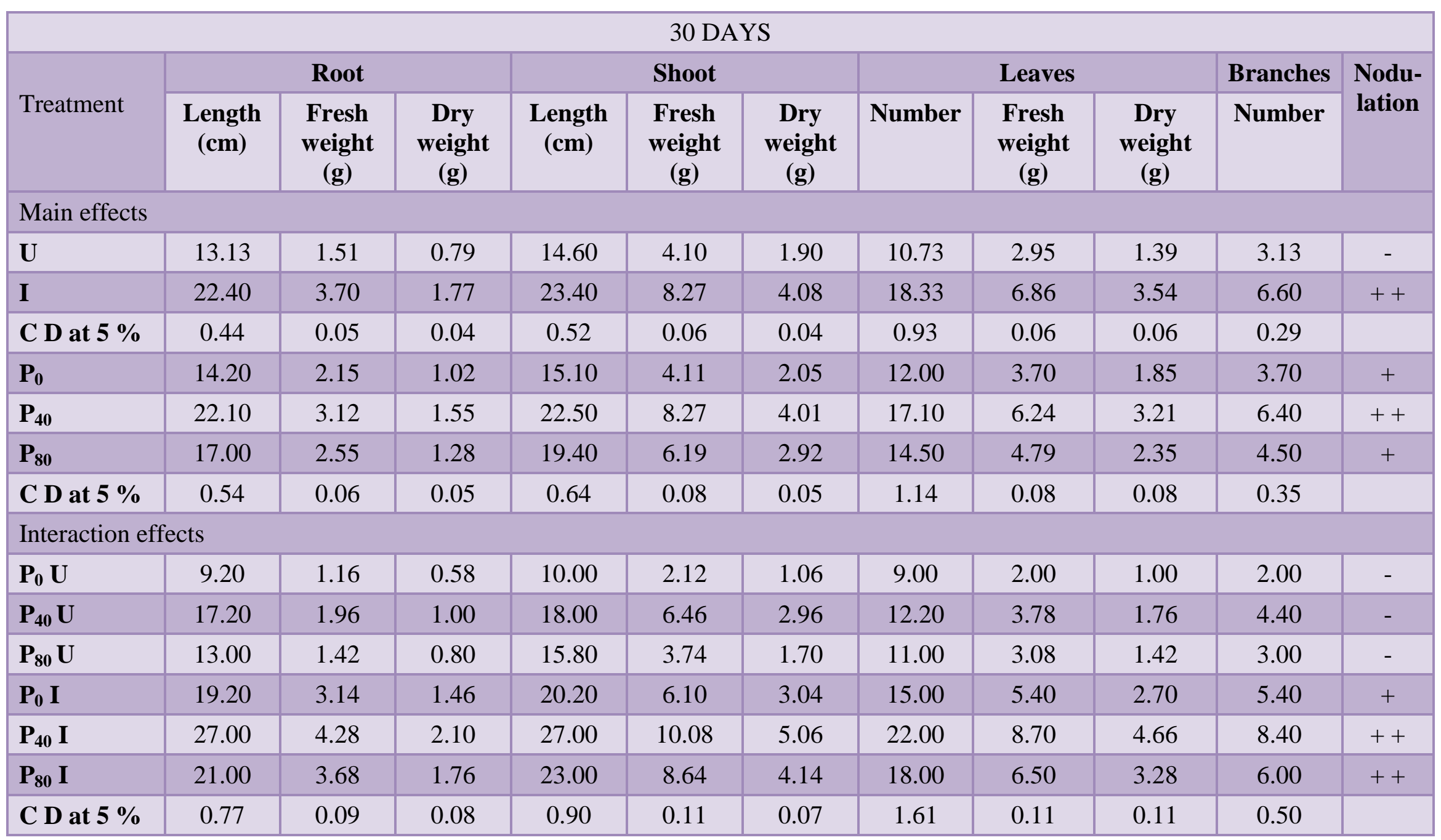

$\mathrm{P}_{0}=$ No phosphorous application; $\mathrm{P}_{40}=40 \mathrm{mg}{ }^{`} \mathrm{P} / \mathrm{kg}$ soil; $\mathrm{P}_{80}=80 \mathrm{mg} ` \mathrm{P} / \mathrm{kg}$ soil; $\mathrm{U}=$ Uninoculated; $\mathrm{I}=$ Inoculated; + = Scanty; + + = Moderate; + + + = Abundant 
Table.4 Effect of Glomus macrocapon inoculation and P amendments on plant growth parameters of Mungbean 45 days after sowing

\begin{tabular}{|c|c|c|c|c|c|c|c|c|c|c|c|}
\hline \multicolumn{12}{|c|}{45 DAYS } \\
\hline \multirow[b]{2}{*}{ Treatment } & \multicolumn{3}{|c|}{ Root } & \multicolumn{3}{|c|}{ Shoot } & \multicolumn{3}{|c|}{ Leaves } & \multirow{2}{*}{$\begin{array}{l}\text { Branches } \\
\text { Number }\end{array}$} & \multirow{2}{*}{$\begin{array}{l}\text { Nodu- } \\
\text { lation }\end{array}$} \\
\hline & $\begin{array}{c}\text { Length } \\
\text { (cm) }\end{array}$ & $\begin{array}{c}\text { Fresh } \\
\text { weight } \\
\text { (g) }\end{array}$ & $\begin{array}{c}\text { Dry } \\
\text { weight } \\
\text { (g) }\end{array}$ & $\begin{array}{l}\text { Length } \\
\text { (cm) }\end{array}$ & $\begin{array}{c}\text { Fresh } \\
\text { weight } \\
\text { (g) }\end{array}$ & $\begin{array}{c}\text { Dry } \\
\text { weight } \\
(\text { g) }\end{array}$ & Number & $\begin{array}{c}\text { Fresh } \\
\text { weight } \\
\text { (g) }\end{array}$ & $\begin{array}{c}\text { Dry } \\
\text { weight } \\
\text { (g) }\end{array}$ & & \\
\hline \multicolumn{12}{|l|}{ Main effects } \\
\hline $\mathbf{U}$ & 21.33 & 2.26 & 1.24 & 26.60 & 7.58 & 3.21 & 17.66 & 6.05 & 2.77 & 5.93 & - \\
\hline $\mathbf{I}$ & 30.33 & 4.88 & 2.44 & 41.93 & 13.20 & 6.38 & 26.33 & 11.00 & 5.35 & 9.93 & +++ \\
\hline C D at $5 \%$ & 0.53 & 0.21 & 0.07 & 0.46 & 0.44 & 0.07 & 0.30 & 0.31 & 0.05 & 0.25 & \\
\hline $\mathbf{P}_{\mathbf{0}}$ & 20.50 & 2.70 & 1.36 & 28.40 & 7.76 & 3.47 & 18.50 & 5.89 & 2.65 & 5.80 & ++ \\
\hline $\mathbf{P}_{40}$ & 31.50 & 4.42 & 2.33 & 40.20 & 13.52 & 6.41 & 25.50 & 12.08 & 5.85 & 10.00 & +++ \\
\hline $\mathbf{P}_{80}$ & 25.50 & 3.60 & 1.84 & 34.20 & 9.90 & 4.52 & 22.00 & 7.61 & 3.69 & 8.00 & ++ \\
\hline C D at $5 \%$ & 0.65 & 0.26 & 0.09 & 0.57 & 0.54 & 0.08 & 0.37 & 0.38 & 0.07 & 0.31 & \\
\hline \multicolumn{12}{|c|}{ Interaction effects } \\
\hline $\mathbf{P}_{\mathbf{0}} \mathbf{U}$ & 17.00 & 1.60 & 0.93 & 21.80 & 4.52 & 2.06 & 15.00 & 4.78 & 1.94 & 4.00 & - \\
\hline $\mathbf{P}_{40} \mathbf{U}$ & 25.00 & 2.84 & 1.60 & 31.00 & 11.04 & 4.66 & 21.00 & 8.16 & 3.88 & 8.00 & - \\
\hline $\mathbf{P}_{80} \mathbf{U}$ & 22.00 & 2.34 & 1.20 & 27.00 & 7.20 & 2.92 & 17.00 & 5.22 & 2.50 & 5.80 & - \\
\hline $\mathbf{P}_{0} \mathbf{I}$ & 24.00 & 3.80 & 1.78 & 35.00 & 11.00 & 4.88 & 22.00 & 7.00 & 3.36 & 7.60 & ++ \\
\hline $\mathbf{P}_{40} \mathbf{I}$ & 38.00 & 6.00 & 3.06 & 49.40 & 16.00 & 8.16 & 30.00 & 16.00 & 7.82 & 12.00 & +++ \\
\hline $\mathbf{P}_{80} I$ & 29.00 & 4.86 & 2.48 & 41.40 & 12.60 & 6.12 & 27.00 & 10.00 & 4.88 & 10.20 & +++ \\
\hline C D at $5 \%$ & 0.92 & 0.36 & 0.13 & 0.80 & 0.76 & 0.12 & 0.53 & 0.54 & 0.10 & 0.44 & \\
\hline
\end{tabular}

$\mathrm{P}_{0}=$ No phosphorous application; $\mathrm{P}_{40}=40 \mathrm{mg} ` \mathrm{P} ` / \mathrm{kg}$ soil $; \mathrm{P}_{80}=80 \mathrm{mg} ` \mathrm{P}$ ' $\mathrm{kg}$ soil $; \mathrm{U}=$ Uninoculated; $\mathrm{I}=\mathrm{Inoculated} ;++=\mathrm{Scanty} ;++=\mathrm{Moderate} ;+++=$ Abundant 
Table.5 Effect of Glomus macrocarpon inoculation and 'P' amendment on plant growth parameters after 60 days of sowing

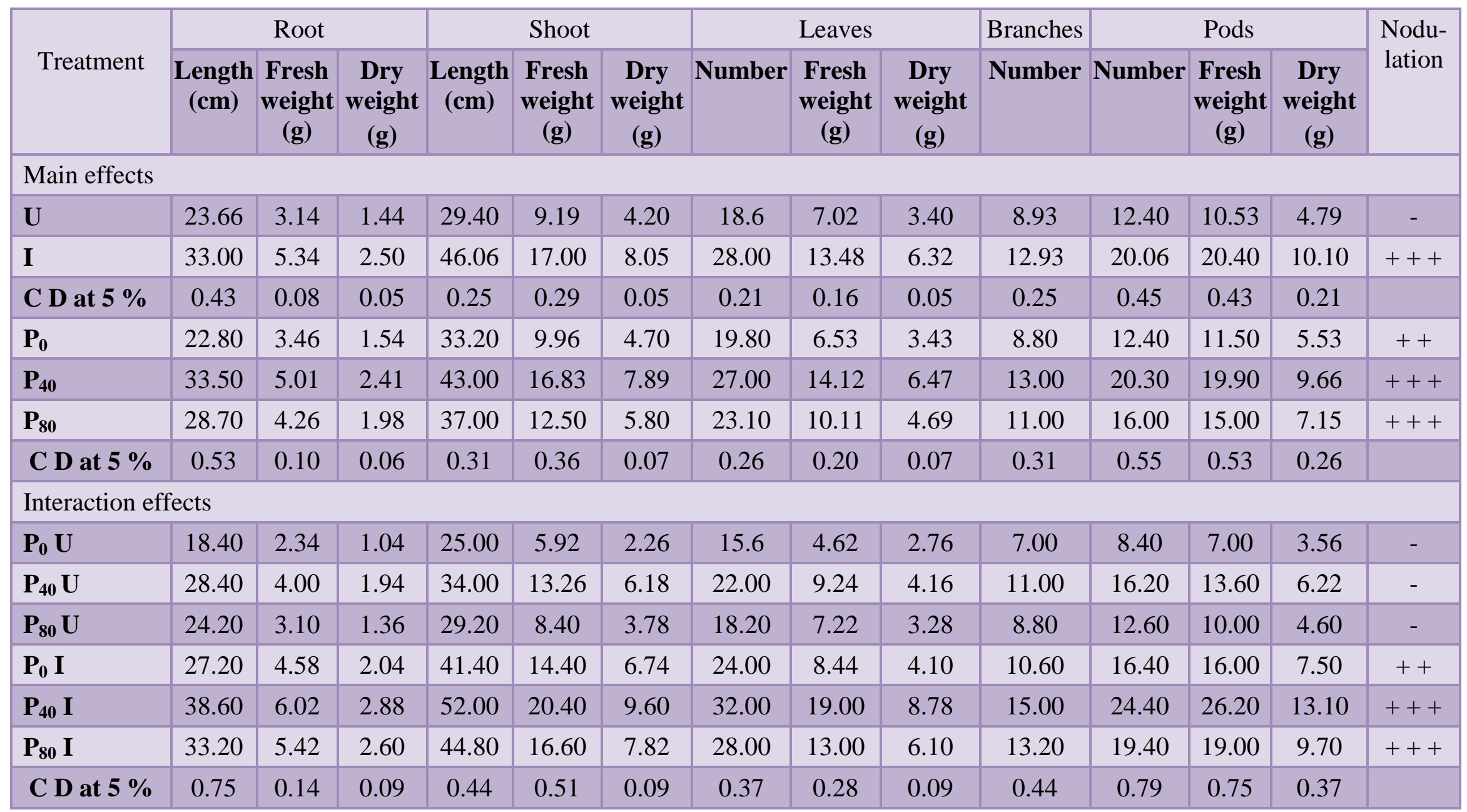

$\mathrm{P}_{0}=$ No phosphorous application; $\mathrm{P}_{40}=40 \mathrm{mg} ` \mathrm{P}^{\prime} / \mathrm{kg}$ soil; $\mathrm{P}_{80}=80 \mathrm{mg} ` \mathrm{P} / \mathrm{kg}$ soil; $\mathrm{U}=$ Uninoculated; $\mathrm{I}=$ Inoculated; + = Scanty; + + = Moderate; + + + = Abundant 


\section{Effect on root nodulation}

Plants exhibited higher nodulation in the mycorrhizal inoculated plants as compared to non-mycorrhizal. Results showed the scanty to moderate level of nodules per plant at the initial stages of plant i.e. after 15 and 30 days of sowing. But with increase in colonization of AM fungi and age of plant, nodulation increased to abundant in after 45 and 60 days of sowing (Table 3-6). $\mathrm{P}$ application also enhanced the nodulation in mycorrhizal inoculated plants. Singh et al., (2017) also observed that the inoculation of Glomus bagyaraji significantly increased the nodulation of chickpea plants.

Plants inoculated with AM fungi showed more number of pods as compared to uninoculated once. It was also noticed that the plants mycorrhizal inoculated plants showed more fresh as we 11 as dry weight along with vigorous pods at $40 \mathrm{mg} \mathrm{P} / \mathrm{kg}$ soil level as compared to 80 and $0 \mathrm{mg} \mathrm{P} / \mathrm{kg}$ soil level. There were 16.40, 24.40 and 19.40 number of pods recorded in AMF inoculated plants at 0 , 40 and $80 \mathrm{mg}$ ' $\mathrm{P}$ '/ $\mathrm{kg}$ soil level after 60 days of sowing. It is suggested that AM fungi enhanced the plant growth as well as yield and yield parameters.

\section{References}

Bargali K (2011) Screening of leguminous plants for VAM association and their role in restoration of degraded lands. J Ame Sci 7: 7-11.

Biermann B and Lindermann R G (1981) Quantifying arbuscular mycorrhizae: A proposed method towards standardization. New Phytol 87: 63-67.

Davis E A and Menge J A (1980) Influence of Glomus fasciculatum and soil phosphorus on Phytophthora root rot of citrus. Phytopathol 70: 447-52.
Gerdemann J W and Nicolson T H (1963) Spores of mycorrhizal Endogone species extracted from soil by

wet sieving and decanting. Trans Br Mycol Soc 46: $235-44$

Harley J L and Smith S E (1983). Mycorrhizal symbiosis. pp 483. Academic Press, London, New York.

Kadian N, Yadav K and Anggarwal A (2014) Application of AM Fungi with Bradyrhizobium japonicum in Improving Growth, Nutrient Uptake and Yield of Vigna radiata L. under Saline Soil. J Stress Physiol Biochem 10: 134-52.

Manke N M, Potdukhe S R, Bramhankar S B and Padghan P R (2008) Effect of rhizobidm and VAM on growth parameters and yield of mungbean. $J \mathrm{Pl}$ Dis Sci 3: 185-88.

Mosse B (1973) Advances in the study of VA mycorrhiza. Ann Rev Phytopath 11: 171-96.

Phillips J M and Hayman D S (1970) Improved procedures for clearing roots and staining parasitic and VA mycorrhizal fungi for rapid assessment of infection. Trans $\mathrm{Br}$ Mycol Soc 55:158-61.

Rabie G H (2005) Influence of arbuscular mycorrhizal fungi and kinetin on the response of mungbean plants to irrigation with seawater. Mycorrhiza 15: 225-30.

Singh N, Singh D and Singh N (2017) Effect of Glomus bagyaraji inoculation and phosphorus amendments on Fusarium wilt of chickpea. Agric Res J 54: 236-43.

Sohrabi M, Mohammadi H and Mohammadi A H (2015) Influence of AM fungi, Glomus mossae and Glomus intraradices on chickpea growth and root rot disease caused by Fusarium solani f.sp. pisi under green house conditions. J Agr Sci Tech 17: 191929.

Souchie E L, Orivaldo J, Saggin-Junior O J, Silva E M R, Campello E F C, Azcon R and Barea J M (2006) Communities of Psolubilizing bacteria, fungi and arbuscular mycorrhizal fungi in grass pasture and secondary forest of Paraty, RJ-Brazil. Ann Acad Bras Cienc 78: 183-93. 


\section{How to cite this article:}

Amandeep Singh, Daljeet Singh and Narinder Singh. 2019. Growth and Yield Response of Mungbean Plants to Colonization of Arbuscular Mycorrhizal Fungi (AMF) (Glomus macrocarpon) and Phosphorus Amendments. Int.J.Curr.Microbiol.App.Sci. 8(12): 3039-3048. doi: https://doi.org/10.20546/ijcmas.2019.812.354 